\title{
Spectroscopy in the Service of Industry*
}

\author{
By J. T. Randall
}

(Research Laboratories of the General Electric Company, Ltd., Wembley, England.)

\section{$\mathrm{I}^{\mathrm{N}}$} recent years, standard spectroscopic technique has been adopted by many industrial research laboratories and has proved a most valuable tool for the identification of the form and nature of impurities which have crept into the materials of ordinary technical processes. It frequently happens that when a manufacturing process goes wrong, there are very few outward and visible signs of the nature of the trouble. A lamp, for example, may have a slightly darkened glass bulb which is indicative of trouble to come. This darkening of the bulb may be due to minute traces, a thin film perhaps, of some deposit. It would be difficult, and sometimes impossible, to determine the nature of the deposit by ordinary chemical means. If the whole of it could be removed it might not woigh more than one ten thousandth of a gram. If the spectroscopist can remove the deposit, he is in an ideal position to give the manufacturer further clues as to the nature of his trouble. Technical processes are also frequently dependent on the addition of a small percentage of some foreign material to the bulk. In applied physics we may mention the quantity of thoria or silica in a tungsten filament; in metallurgy the percentage of nickel or tungsten in a steel.

The methods of excitation of the spectra are usually the standard ones of arc and spark; sometimes the technique of absorption spectra may be used. A very useful modification of the spark method consists in 'exploding' a wire and exciting the spectrum of the resultant vapour. This was developed by $J$. W. Rydet, and is of great value in the electrical industry where the quality of wire is such an important factor. In order to facilitate the analysis of unknown spectra, Ryde and Jenkins have developed a special powder containing more than fifty elements. The quantities have been so adjusted that the raies ultimas of all of these elements appear when the powder is 'arced'. This method and the 'ratioquantitative' method of Judd Lewis have been of great assistance in the routine applications of spectroscopy to industrial problems.

The applications of the general spectroscopic method are many, and it is impossible to refer to

* Summary of a lecture given before Section Af (Technical Physics) of the British Association at Leicester on September 8 .

+ Research Laboratories of the General Electric Co. Ltd. more than a few here. The exploded wire method has been used with great success on small samples of various metallic wires such as tungsten, nickel and molybdenum. The transmission of ultra-violet light through glass is dependent, among other things, on the quantity of iron present. Preparation of a set of standards containing, say, 0.005-0.05 per cent of $\mathrm{Fe}_{2} \mathrm{O}_{3}$, enables the quantity of iron in various glassmaking sands to be estimated. The testing of steels before they leave the works is now carried out by means of the 'steeloscope'. An upright rod of pure iron comes into contact with the test bar at some convenient point and the arc spectrum is observed. Bars which may have got into the consignment by mistake can in this way be readily identified. This is one of the first instances in which the spectroscope has been used in a routine manner by unskilled workers. Spectroscopic technique has been of great value in the development of hot cathode discharge tubes, such as the Osira lamp. In work of this kind new fundamental information is frequently obtained. With the Osira lamp, changes of colour and intensity can be followed as the pressure of mercury vapour increases: similar effects are to be observed with thallium rare gas spectra, as Krefft has shown.

The work of Hevesy and Laby on X-ray spectra is likely to be of use to industry, but has not yet received wide application. On a broad view, the applications of X-ray diffraction principles may be included in this summary. Provided the crystal structure is known, the X-ray crystallographer can indicate the form of any particular substance. For example, the state of the silica in refractory materials has a considerable effect on the expansion properties. The properties of drawn wires, in respect of crystal orientation, can readily be studied by this method. The cause of the red colour of selenium ruby glasses has been shown to be due to the actual composition of a cadmium selenide-cadmium sulphide solid solution. X-ray investigation of commercial glasses and of carbon blacks has also given useful information. Violet phosphorus has been shown to be fundamentally the same as red phosphorus, the only difference being one of crystal size. Very interesting work on the nature of oxide-coated cathodes has been correlated with their thermionic properties.

\section{Natural Colouring Matters}

T $N$ opening a discussion on September 7 in Section B (Chemistry) of the British Association meeting at Leicester, which followed Prof. R. Robinson's address to the Section on "Natural Colouring Matters and their Analogues", Prof. R. Kuhn first reviewed the inter-relationship of the colouring matters of the carotene group. These substances are synthesised in plants and their molecules each contain forty carbon atoms. They undergo two types of degradation : in the animal body they produce vitamin A by hydrolytic fission, whilst in plants they are oxidised to carotenoids containing fewer carbon atoms. Among the natural oxidation products so formed are the pigments bixin, crocetin and azagrin. The other decomposition products formed in these oxidations frequently possess characteristic colour, smell or taste. Similar oxidations of carotene have recently been carried out in the laboratory.

Another widely distributed class of natural dyes is the flavines. Two of these, ovoflavine from eggalbumin and lactoflavine from milk, have recently been obtained as yellow erystalline solids and are probably identical. These substances exhibit high vitamin $B_{2}$ activity, which persists after repeated crystallisation and even after acetylation and hydrolysis of the acetyl derivative. The flavines appear to be necessary items in the diet of mammals, like the carotenes. The general properties of the two classes 